\section{THU0501 EARLY DIAGNOSIS OF THE AUTOIMMUNE LYMPHOPROLIFERATIVE SYNDROME (ALPS) IN PATIENTS WITH UNDEFINED AUTOINFLAMMATORY OR AUTOIMMUNE DISORDERS: THE PRACTICAL ROLE OF A FLOW CYTOMETRY PANEL}

C. Matucci Cerinic ${ }^{1,2}$, L. Oliveira Mendonca ${ }^{3}$, M. Miano ${ }^{4}$, P. Terrnaova ${ }^{4}$, F. Casabona ${ }^{2}$, M. Bustaffa ${ }^{1,2}$, F. Bovis ${ }^{5}$, R. Caorsi ${ }^{1,3}$, S. Volpi ${ }^{1,3}$, A. Ravelli ${ }^{1}$, C. Dufour ${ }^{4}, M$. Gattorno ${ }^{3}$ on behalf of Caterina Matucci Cerinic and Leonardo Oliveira Mendonca equally contributed to the work. ${ }^{1} I R C C S$ Istituto G. Gaslini, Genoa, Italy, Clinica Pediatrica e Reumatologia, Genova, Italy; ${ }^{2}$ IRCCS IStituto Giannina GGaslini, Genova, DINOGMI, Università degli Studi di Genova, Genova, Genova, Italy; ${ }^{3}$ IRCCS Istituto G. Gaslini, Genoa, Italy, Centro per le Malattie Autoinfiammatorie ed Immunodeficienze, Clinica Pediatrica e Reumatologia, Genova, Italy; ${ }^{4}$ IRCCS Istituto Giannina Gaslini, Genova, Clinical and Experimental Haematology Unit, Department of Haematology/Oncology, Genova, Italy; ${ }^{5}$ University of Genova, Department of Health Sciences (DISSAL), Section of Biostatistics, Genoa, Italy,

Genova, Italy

Background: ALPS is a rare disorder due to a defective apoptotic mechanism leading to abnormal lymphoproliferation and autoimmunity. The disease is difficult to identify in the early phase when it may be misdiagnosed. Elevated TCR alpha-beta CD4-CD8- lymphocytes (double negative T lymphocytes DNT) together with hyperlgG, high levels of IL10, $\| 18$, vitamin B12 and soluble Fas ligand have been suggested as the main ALPS hallmarks (1). Therefore, a specific flow cytometry panel (DNT cells, ratio of CD25+CD3+ to HLA-DR+CD3+ cells, increased B220+ T-cells, and decreased CD27+ memory $B$ cells) has been proposed to serve as a diagnostic screen for ALPS (2).

Objectives: To evaluate the usefulness of a specific lymphocyte flow cytometry panel in the early identification of ALPS/ALPS-like disorders in a cohort of patients with undefined autoinflammatory or autoimmune disorders.

Methods: The clinical data of patients referred to the pediatric Rheumatology Unit of the Istituto Giannina Gaslini Hospital for a suspicion of autoimmune or autoinflammatory condition from October 2015 to April 2018, were retrospectively analyzed. Data on clinical manifestations, laboratory workup, genetic analysis and treatment were collected. Flow cytometry was included among the screening panel: DNT, CD25+CD3+, HLA-DR+CD3+ cells, B220+ T-cells, and CD27+ memory B cells were included. A statistical analysis was performed: data were analyzed with an univariate logistic regression analysis, to identify the most significant variables associated with ALPS. These variables were then included in a multivariate analysis to select a set of clinical and laboratory parameters, each of them associated with a significant probability to be associated with ALPS independently from other variables.

Results: 475 patients were retrospectively analized. 211 patients not fulfilling the inclusion criteria were excluded. The patients were classified as follows: i) Autoimmune disease 26 pts (10 SLE; 3 MCTD; 6 jDM; 5 Behçet; 1 SjS; 1 Kawasaki) ii) Juvenile Idiopathic Arthritis 35 pts iii) Monogenic systemic autoinflammatory disease (MSAID) 27 pts (17 FMF; 3 MKD; 1 TRAPS; 4 DADA2; 2 SAVI) iv) PFAPA 100 pts v) Systemic Undefined Recurrent Fever 45 pts vi) Undetermined-SAID 15 pts vii) ALPS/ ALPS probable 16 pts. The flow cytometry panel showed, as expected, an elevation of DNT in all ALPS patients. Among the other parameters, CD3CD25+/CD3HLADR+, and B220+ T cells, were significatively altered in $75 \%$ of ALPS patients. Conversely, B CD27+ did not differentiate ALPS from the other subgroups. The multivariate analysis revealed 5 clinical/laboratory parameters that showed the higher independent association to ALPS in the cohort of patients. Splenomegaly, female gender, elevated DNT, arthralgia and elevated alfabeta+B220+ lymphocytes were positively and significantly associated to ALPS.

Conclusion: The use of the specific flow cytometry panel, comprehensive of DNT, B220+, HLA-DR and CD25, in patients with undefined autoinflammatory or autoimmune disorders may identify a subgroup of patients with ALPS.

References:

[1] Joao B. Oliveira et al. Blood 2010; 116 (14): e35-e40.

[2] Lenardo MJ et al. Immunity. 2010;32(3):291-295.

Disclosure of Interests: Caterina Matucci Cerinic: None declared, Leonardo Oliveira Mendonca: None declared, maurizio miano: None declared, paola terrnaova: None declared, federica casabona: None declared, Marta Bustaffa: None declared, Francesca Bovis: None declared, Roberta Caorsi: None declared, Stefano Volpi: None declared, Angelo Ravelli: None declared, Carlo Dufour: None declared, Marco Gattorno Consultant of: Sobi, Novartis, Speakers bureau: Sobi, Novartis

DOI: 10.1136/annrheumdis-2020-eular.3795

\section{THU0502 \\ EFFICACY AND SAFETY OF SECUKINUMAB TREATMENT IN JUVENILE IDIOPATHIC ARTHRITIS PATIENTS}

I. Kriulin ${ }^{1,2}$, E. Alexeeva ${ }^{1,2}$, T. Dvoryakovskaya ${ }^{1,2}$, K. Isaeva $^{1}$, A. Chomakhidze ${ }^{1}$, E. Chistyakova ${ }^{1,2}$, O. Lomakina ${ }^{1}$, R. Denisova ${ }^{1}$, A. Mamutova ${ }^{1}$, A. Fetisova ${ }^{1}$, M. Gautier ${ }^{1}$, D. Vankova ${ }^{1}$, E. Krekhova ${ }^{2}$, M. Shingarova ${ }^{2}$, A. Alshevskaya ${ }^{3}$, A. Moskalev ${ }^{3} .{ }^{1}$ National Medical Research Center of Children's Health, Moscow, Russian Federation; ${ }^{2}$ Sechenov First Moscow State Medical University (Sechenov University), Moscow, Russian Federation; ${ }^{3}$ Biostatistics and Clinical Trial Center, Moscow, Russian Federation

Background: Anti-IL-17A biologic drug secukinumab (SEC) proved to be effective for treatment of psoriatic arthritis. However data about its efficacy in juvenile idiopathic arthritis (JIA) are restricted to off-label experience.

Objectives: To evaluate the effectiveness and safety of SEC in JIA patients in the National Medical Research Center of Children's health, Moscow, Russia.

Methods: 25 patients started SEC therapy from 12/2017 to 11/2019 in single-center prospective study. 3 patients withdrew treatment: two patients $(8 \%)$ due to AE (1 - allergy followed by MAS after first injection and 1 - leukopenia) and one patient $(4 \%)$ - after 10 months of treatment due to secondary inefficacy. Among others, 14 patients which were successfully treated for 6 months or longer were included into analysis. At the baseline, information was collected on the characteristics of the onset of the disease, previous therapy and its success. Patients were monitored at least 1 time per year. At each visit, clinical and laboratory characteristics of JIA severity were assessed. Response to therapy was assessed using the ACRPedi 30/50/70/90 criteria, the C.Wallace criteria for inactive disease (WID) and clinical remission. AEs were assessed at each visit.

Results: Among 14 patients received SEC for at least 6 months, $7(50 \%)$ have enthesitis-related arthritis, one (7.1\%) - persistent oligoarthritis, $4(28.6 \%)$ RF-negative polyarthritis, $2(14.3 \%)$ - psoriatic arthritis. 6 patients (42.9\%) were HLA-B27 positive. Median age of JIA onset was 8.8 (IQR 5:11), age at SEC initiation - 14 (9.9:16.1), disease duration before SEC start - $3.3(2.7: 5.8) .7(50 \%)$ were biologics-naïve, $2(14.3 \%)$ were previously treated with anti-TNF drug, 5 $(35.7 \%)$ have 2 or more different biologics in anamnesis.

SEC demonstrated high efficacy after the first injection resulting in JADAS-71 decreasing in all patients by median $4.3(1.6: 7.1)$ points and 7/7/5/2 patients (50\%/50\%/35.7\%/14.3\%) achieved ACR Pedi 30/50/70/90 response.

After 6 months of treatment, WID was achieved by $7(50 \%)$ patients, JADAS71 decreased from baseline level $15.2(12.7: 20.5)$ to $0.8(0: 4.2)$ points, and 14/13/11/9 patients (100\%/92.9\%/78.6\%/64.3\%) achieved ACR Pedi 30/50/70/90 response. One patients who had active uveitis at SEC initiation remained with subactive uveitis; one patient with uveitis remission had not flare episodes during follow-up period. One patient $(7.1 \%)$ had successfully treated evaluation of transaminases after 4-th injection.

Conclusion: Secukinumab showed high effectiveness and safety in children with JIA and can be further used both as a first-line drug in JIA associated with HLA-B27, and as an alternative drug for the ineffectiveness of the standard treatment regimen with biologics. No serious adverse events were registered during follow-up period.

Disclosure of Interests: Ivan Kriulin: None declared, Ekaterina Alexeeva Grant/ research support from: Roche, Pfizer, Centocor, Novartis, Speakers bureau: Roche, Novartis, Pfizer., Tatyana Dvoryakovskaya: None declared, Ksenia Isaeva: None declared, Aleksandra Chomakhidze: None declared, Evgeniya Chistyakova: None declared, Olga Lomakina: None declared, Rina Denisova: None declared, Anna Mamutova: None declared, Anna Fetisova: None declared, Marina Gautier: None declared, Dariya Vankova: None declared, Elizaveta Krekhova: None declared, Meyri Shingarova: None declared, Alina Alshevskaya: None declared, Andrey Moskalev: None declared

DOI: 10.1136/annrheumdis-2020-eular.5847

\section{THU0503 PLUTO TRIAL: SENSITIVITY ANALYSES OF SRI4 RESPONSE WITH BELIMUMAB VS PLACEBO IN PAEDIATRIC PATIENTS WITH CHILDHOOD-ONSET SYSTEMIC LUPUS ERYTHEMATOSUS (CSLE)}

N. Ruperto ${ }^{1}$, H. Brunner ${ }^{2}$, M. Mori ${ }^{3}$, J. Clinch ${ }^{4}$, R. Syed ${ }^{5}$, N. Iwata ${ }^{6}$, D. Bass ${ }^{7}$, B. Ji ${ }^{8}$, A. Hammer ${ }^{7}$, M. Okily ${ }^{8}$, G. Eriksson ${ }^{7}$, H. Quasny ${ }^{9} .{ }^{1}$ Istituto Giannina Gaslini, Genoa, Italy; ${ }^{2}$ Cincinati Children's Hospital, Cincinnati, United States of America; ${ }^{3}$ Tokyo Medical and Dental University, Tokyo, Japan; ${ }^{4}$ University Hospitals Bristol, Bristol, United Kingdom; ${ }^{5}$ St Louis University, St Louis, United States of America; ${ }^{6}$ Aichi Children's Health and Medical Center, Obu, Japan; ${ }^{7}$ GlaxoSmithKline, Collegeville, United States of America; ${ }^{8}$ GlaxoSmithKline, Uxbridge, United Kingdom; ${ }^{9}$ GlaxoSmithKline, Research Triangle Park, United States of America 